\title{
Modelling Dynamic Response of Hydraulic Fluid Within Tapered Transmission Lines
}

\author{
Jeremy ven der Buhs and Travis Wiens \\ Department of Mechanical Engineering, University of Saskatchewan, Saskatoon, Saskatchewan, Canada \\ E-mail: jeremy.venderbuhs@usask.ca,travis.wiens@usask.ca
}

\begin{abstract}
This paper examines modelling the laminar dynamic fluid responses within hydraulic transmission lines that have a tapered shape from the inlet to outlet. There are excellent models available for fast simulation of pressure and flow dynamics within uniform lines, however the established models for tapered lines have some notable inaccuracies and simulation complexities. The transmission line method (TLM) structure is applied in this paper since it can be computed quickly and has shown to accurately model the effects of frequency-dependent friction. This paper presents a method of optimizing the TLM weighting functions, minimizing the error between the TLM transmission matrix terms and a numerical exact solution. Optimizations have shown that using the TLM to model tapered lines has improved accuracy over the existing models when compared in the frequency domain. Two-dimensional interpolation of a look-up table is possible allowing for quick selection of the optimized parameters. This model can be used in numerous applications where line dynamic effects must be accounted for, especially with digital hydraulic switched inertance converters.
\end{abstract}

Keywords: Transmission line method, TLM, tapered fluid lines, optimization, modelling, simulation.

\section{Introduction}

Computer modelling of fluid transmission lines of constant cross-sectional area is an area of hydraulics where extensive research has been performed previously as well as very recently [1-7]. However, the established research into the modelling of tapered transmission lines has some shortcomings which requires further study and refinement. Time domain models describing line dynamics are greatly desired as it allows for simple implementations with time domain numerical solvers, and interfaces well with nonlinear component models such as valves and accumulators. The ability to model pressure and flow transients within fluid lines is important for predicting the performance of hydraulic systems, especially those where line effects should be considered.

As shown in fig. 1, the transmission line method (TLM) is a method of modelling which uses a network of linear transfer functions and time delays to model wave propagation [1], and has had recent enhancements accurately modelling the effects of frequency-dependent friction, all while solving within the time domain [2,3]. While the TLM currently works for uniform lines of constant cross-section, it has not yet been applied to tubes of varying cross-section. The first mention of modelling tapered lines employs a method of characteristics (MOC) solution to the differential equations, however, the proposed MOC solution was only used and analyzed for a uniform line [4]. An approximated analytical solution for

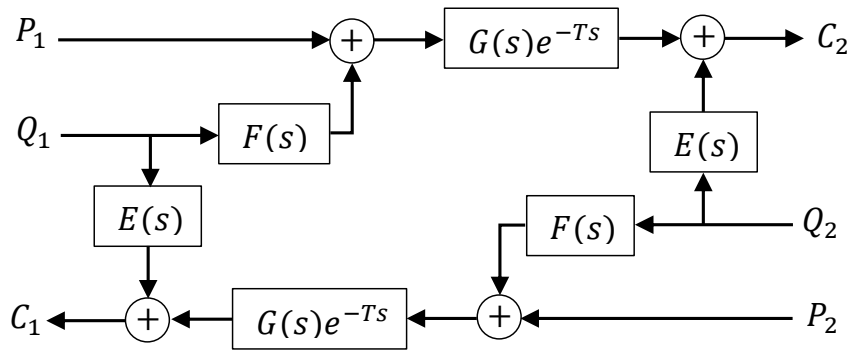

Figure 1: Block diagram of the TLM [1].

frequency dependent flow through tapered lines was derived in [5]. The approximate transmission matrix they derived was taken further in [6] by applying modal analysis (MA) and rational polynomial transfer function approximations (RPTFA) of the transcendental functions in the solution, allowing for simulations to be performed in the time domain. No further research into modelling tapered lines has appeared in the literature until this point, and the need for a robust, fast, and accurate model for tapered transmission lines has heightened with the growth in switched inertance converter research [8].

This paper will look at modelling tapered fluid lines using the TLM model and optimization procedure similar to that in [3] where weighted transfer functions make up the $E, F$, and $G$ transfer functions. The equations and optimization procedure 
are described in detail. Frequency domain results will be analyzed, and comparisons will be made between previous models, the exact solution, and the proposed model. Also, time domain simulations of the model will be discussed.

\section{Previous Models}

The differential equations that describe one-dimensional flow, $Q$, and pressure, $P$, through the tapered tube shown in fig. 2 are the equations of motion and continuity, expressed in the Laplace domain, respectively [7]:

$$
\begin{gathered}
\frac{d P(x, s)}{d x}+\frac{\rho s}{A(x)} Q(x, s) N(x, s)=0 \\
P(x, s)=-\frac{\partial Q(x, s)}{\partial x} \frac{K}{A(x) s}
\end{gathered}
$$

where $\rho$ is the fluid density, and $s$ is the Laplace operator. $N$ is the frequency-dependent friction term, commonly used in previous research defined as [1-7]

$$
N(x, s)=-\frac{J_{0}\left(j r(x) \sqrt{\frac{s}{v}}\right)}{J_{2}\left(j r(x) \sqrt{\frac{s}{v}}\right)} .
$$

Frequency-dependent friction has shown to be a realistic phenomenon, and models that implement it have shown to be significantly more accurate than models that use steady friction. Since the proposed model natively uses a summation of weighting functions, it can be expected that there would be negligible computational time savings if steady friction were considered. As such, frequency-dependent friction will be exclusively considered in this paper.

Within a tapered transmission line, the radius varies linearly from inlet to outlet given by eq. (4), and is visualized in fig. 2.

$$
r(x)=r_{1}+\frac{\left(r_{2}-r_{1}\right) x}{l}
$$

The existing model for modelling tapered fluid lines was developed in $[5,6]$. The transmission matrix, eq. (5) and (6), is not an exact solution, but rather an approximated analytical solution to the set of ordinary differential equations previously defined. Within its derivation, higher order terms of a Taylor series expansion were rejected in order to allow the

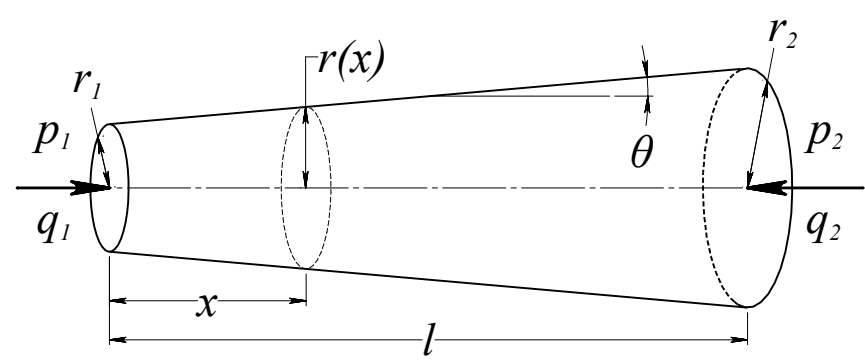

Figure 2: Schematic of a tapered transmission line. possibility of a closed form solution in the frequency domain. This simplification creates notable inaccuracy at higher frequencies.

$$
\left(\begin{array}{c}
P_{1} \\
Q_{1}
\end{array}\right)=\left(\begin{array}{cc}
\cosh \Gamma-\frac{\xi \lambda_{o}}{D S \sqrt{N}} \sinh \Gamma & Z_{c} \sqrt{N} \sinh \Gamma \\
\frac{1}{Z_{c} \sqrt{N}} \sinh \Gamma & \cosh \Gamma+\frac{\xi \lambda_{o}}{D S \sqrt{N}} \sinh \Gamma
\end{array}\right)\left(\begin{array}{c}
\frac{P_{2}}{1-\xi \lambda_{o}} \\
\frac{Q_{2}}{1+\xi \lambda_{o}}
\end{array}\right)
$$

where,

$$
\begin{gathered}
\Gamma=D S \gamma_{o}\left[1+\xi\left(1-\lambda_{o}\right)\right], D=\frac{v l}{c r_{1}^{2}}, Z_{c}=\frac{\rho c \gamma_{o}}{\pi r_{1}^{2}}, \\
\lambda_{o}=\frac{1}{2}\left[1-\left\{\frac{\chi_{o}\left(\gamma_{o}^{2}-1\right)}{2 \gamma_{o}}\right\}^{2}\right], t_{o}=\frac{r_{1}^{2}}{v}, S=s t_{o}, \\
\gamma_{o}=\left[-\frac{J_{o}(j \sqrt{S})}{J_{2}(j \sqrt{S})}\right], \chi_{o}=\sqrt{S}, \xi=\frac{\theta l}{r_{1}} .
\end{gathered}
$$

This model is presented in nondimensional form, with parameters nondimensionalized with respect to the inlet radius, $r_{1}$. In its current form, this transmission matrix cannot be solved in the time domain, a similar problem that previous researchers have encountered with simulating transmission lines. Further approximation is necessary in order to solve this system of equations in the time domain. In [6], modal analysis (MA) and rational polynomial transfer function approximations (RPTFA) were performed in order for the transmission matrix to be solved in the time domain. While these approximations, tabulated in their paper, show good agreement with the approximate transmission matrix proposed by [5], it is important to note that the transmission matrix is an approximated closed form solution, and has some notable discrepancies to the exact analytical solution, and has a very limited range of accuracy.

In order for a true two-port (inlet and outlet), 4-terminal (pressure and flow at each end) transmission line model to work accurately it needs to provide symmetrical solutions, meaning that given the same boundary conditions and dimensions, the transfer function must be the same if the ends are exchanged. Consider 2 cases, Case 1 has a diverging tube with a blocked outlet, and Case 2 has a converging tube with a blocked inlet. In both cases, the blocked port has the larger radius, and the smaller radius is open. By maintaining the same dimensions, the pipe in Case 2 is simply a flipped version of Case 1 . The transfer functions in eq. (5) and eq. (6) were calculated and are compared in fig. 3. The logical result of this study should have shown the transfer functions be exactly the same, however there is a noticeable shift in the frequency peaks. The same study was performed using the exact solution calculated numerically from the set of ordinary differential equations previously defined, and showed that, in this case, the results are the same. This asymmetry in the approximate transmission matrix leads to significant inaccuracy when implemented in the time domain.

Another issue with the existing transmission matrix is inaccuracies at higher harmonics. Figure 3 shows how the 


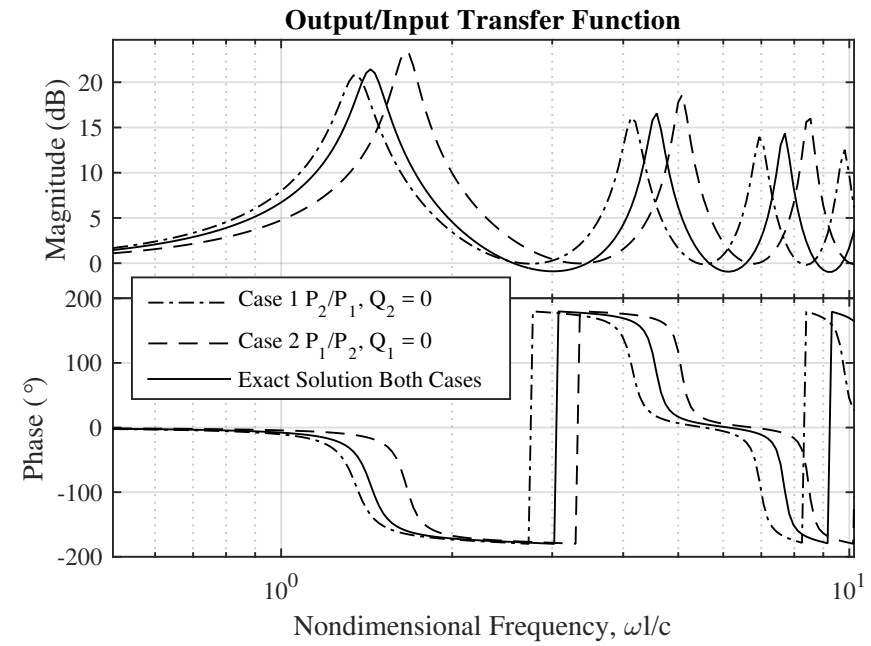

Figure 3: Transfer function asymmetry and comparison to the exact numerical solution.

$P_{2} / P_{1}$ transfer function for a blocked outlet compares from the transmission matrix solution to the exact numerical solution. While the first resonance is modelled closely, higher resonances of significant power are not closely matched. This difference becomes greater with increased transmission line taper.

\section{Proposed Model}

The proposed model is similar to the one developed for uniform transmission lines in [3]. The 2-port model relating pressure and flow at the inlet, to the pressure and flow at the outlet is given by the transmission matrix in eq. (7) [3]. The characteristics $C_{1}$ and $C_{2}$ are related to pressure and flow by eq. (8) and (9) [1]. The characteristic impedance of the tapered line is $Z_{c}$.

$$
\begin{gathered}
\left(\begin{array}{c}
P_{1} \\
Q_{1} Z_{c}
\end{array}\right)=\left(\begin{array}{ll}
t_{11} & t_{12} \\
t_{21} & t_{22}
\end{array}\right)\left(\begin{array}{c}
P_{2} \\
Q_{2} Z_{c}
\end{array}\right) \\
P_{1}=C_{1}+Z_{c} Q_{1} \\
P_{2}=C_{2}+Z_{c} Q_{2}
\end{gathered}
$$

For a tapered line, the characteristic impedance can be derived using the definition in [9] where wave propagation time is divided by the hydraulic capacitance of the pipeline. The volume of a tapered pipeline is that of a right circular cone frustum, and is included in the calculation of hydraulic capacitance. Therefore:

$$
Z_{c}=\frac{3 \rho c}{\pi r_{\max }^{2}}\left(1+\lambda+\lambda^{2}\right)^{-1}
$$

where $\lambda$ is the taper ratio, which is simply defined as the minimum pipe radius divided by the maximum pipe radius:

$$
\lambda=\frac{r_{\min }}{r_{\max }} .
$$

This parameter is the same regardless if the pipe is converging or diverging in shape. The steady state resistance of a tapered transmission line, $R$, is defined by integrating uniform
Hagen-Poiseuille pressure drops over the entire length of the pipeline. Lubrication theory is employed since the change in radius over a small element length $d x$ is considerably smaller than the size of the pipe itself. The hydraulic resistance is then found to be:

$$
R=\frac{8 \rho v l}{\pi r_{\max }^{4}}\left[\frac{1+\lambda+\lambda^{2}}{3 \lambda^{3}}\right]
$$

Having obtained expressions of characteristic impedance and resistance, the dissipation number, $\beta$, for a tapered transmission line can be found using the following relationship:

$$
\beta=\frac{R}{8 Z_{c}}=\frac{v l}{c r_{\max }^{2}}\left[\frac{\left(1+\lambda+\lambda^{2}\right)^{2}}{9 \lambda^{3}}\right] .
$$

It is important to note that for a pipeline of uniform cross section, the taper ratio would be 1 , and the resulting characteristic impedance, laminar resistance, and dissipation number would reduce to the same as used in the previous models for uniform tubes [2,3].

The TLM structure contains linear transfer functions $E, F$, and $G$ which are arranged in the configuration shown in fig. 1 and are defined by the weighting functions as follows [3].

$$
\begin{gathered}
E(s)=Z_{c} \sum_{i=1}^{k} \frac{m_{E i}}{n_{i}+T s} \\
F(s)=Z_{c}+b E(s) \\
b=1-\frac{8 \beta}{\sum_{i=1}^{k} \frac{m_{E i}}{n_{i}}} \\
G(s)=1-\sum_{i=1}^{k} \frac{m_{G i} T s}{n_{i}+T s} \\
T^{\prime}=\tau T=\tau \frac{l}{c}
\end{gathered}
$$

The weighting factors $m_{E i}, m_{G i}$, and $\tau$ are found using a constrained optimization which is explained in the following section. Here, the $F$ transfer function is scaled off of the $E$ transfer function. This gives the model the ability to accurately compute the pressure drop during steady state conditions. The weighting factors $n_{i}$, are the same as in the series proposed in [3], which are given as follows:

$$
n_{1}=\frac{0.3}{1+3 \beta}, \quad n_{i+1}=3 n_{i}
$$

Using separate weighting factors for $F$ was also investigated, but it provided no significant increase in accuracy, and added considerable time to the optimization procedure. Similar to [3], better results were obtained by slightly modifying the wave propagation time $T$ by a factor, $\tau$. From the TLM structure and applying some algebra, the transmission matrix terms can be found as follows:

$$
t_{11}^{*}=\frac{\left(E+Z_{c}\right) G^{-1} e^{j \omega T^{\prime}}+F G e^{-j \omega T^{\prime}}}{E+Z_{c}+F}
$$




$$
\begin{gathered}
t_{12}^{*}=\frac{\left(E+Z_{c}\right)^{2} G^{-1} e^{j \omega T^{\prime}}-F^{2} G e^{-j \omega T^{\prime}}}{E+Z_{c}+F} \\
t_{21}^{*}=\frac{G e^{-j \omega T^{\prime}}-G^{-1} e^{j \omega T^{\prime}}}{E+Z_{c}+F} \\
t_{22}^{*}=-t_{11}^{*} .
\end{gathered}
$$

Unlike uniform hydraulic transmission lines, tapered lines are asymmetrical in their geometry. This lead to the investigation of using different transfer functions for the inlet and outlet in order to see if there was an increase in accuracy of the TLM approximation. The following scenarios were examined:

- Separate $G$ transfer functions with separate weighting factors. Resulted in no increase in accuracy, and increased optimization effort.

- Separate wave propagation times $T^{\prime}$ for either direction. Resulted in increased error.

- Separate $E$ transfer functions, with the corresponding $F$ transfer function scaled from it. Resulted in no increase in accuracy, and increased optimization effort.

- Separate $E$ and $F$ transfer functions where $F$ is not scaled off of $E$. Resulted in significant error in magnitude and phase, as well as substantial increase in optimization effort.

These investigations showed no increase in accuracy over the standard TLM configuration, and all resulted in longer computation time, which was not desirable.

\section{Optimization Algoritm}

As with all optimization problems, an objective function to be minimized must be defined. In [3], Johnston et al. define an objective function for a uniform tube that minimizes the error in $t_{12}$ and $t_{21}$ in eq. (21) and (22), but ignores $t_{11}$ and $t_{22}$. Here, the objective function, $f$, similar to the function used in [3], is used but with the addition of the $t_{11}$ and $t_{22}$ terms. This objective function attempts to minimize the error between the exact transmission matrix and the TLM approximated transmission matrix.

$$
\begin{gathered}
f=\sum_{0.01 \leq \omega T \leq n_{k}} \frac{\left|\frac{t_{12}-t_{12}^{*}}{Z_{c}}\right|^{2}}{\omega T}+\sum_{0.01 \leq \omega T \leq n_{k}} \frac{\left|\left(t_{21}-t_{21}^{*}\right) Z_{c}\right|^{2}}{\omega T}+ \\
\sum_{0.01 \leq \omega T \leq n_{k}} \frac{\left|\left(t_{11}-t_{11}^{*}\right)\right|^{2}}{\omega T}+\sum_{0.01 \leq \omega T \leq n_{k}} \frac{\left|\left(t_{22}-t_{22}^{*}\right)\right|^{2}}{\omega T}+\varepsilon_{E}+\varepsilon_{G} \\
\varepsilon_{E}=\sum_{i=3}^{k}\left[\max \left(0, m_{E i}-3 m_{E i-1}\right)\right]^{2} \\
\varepsilon_{G}=10\left[\max \left(0, \sum_{i=1}^{k}\left(m_{G i}\right)-1\right)\right]^{2}
\end{gathered}
$$

In this case, the transmission matrix terms denoted with an asterisk represent the approximation from the TLM, while the terms without the asterisk represent the numerical solution yielded from solving the boundary value problem of eq. (1) and (2). The terms $\varepsilon_{E}$ and $\varepsilon_{G}$ are the same soft constraints presented in [3]. The optimization was calculated with 50 points per decade over the frequency range of $0.01 \leq \omega T \leq n_{k}$.

The optimization procedure first required the exact solution of eq. (1) and (2). The exact solution is calculated numerically using MATLAB $R$ and its bvp4c boundary value problem solver. The exact solution is the transmission matrix in eq. (7) for a given tapered tube. By setting $Q_{2}$ to $0, t_{11}$ and $t_{21}$ can be found. Then by setting $P_{2}$ to $0, t_{12}$ and $t_{22}$ can be calculated. It is important to note that solving this boundary value problem does add significant computation time to the optimization procedure. However, computation time can be reduced when the problem is solved in parallel, and with more available cores, the problem becomes faster to solve. Also, the boundary value problem only needs to be solved once for each parameter set. After computing the numerical solution, the optimization of eq. (24) is performed using the fmincon function in MATLAB $R$, where the only set constraint was a lower bound of 0 on the parameters. This ensures the parameters are all positive, similar to the constraint in [3]. The results for a uniform tube given in $[3,10]$ were used as an initial guess for the iterative solver. Using this algorithm and initial guess does not necessarily find the global minimum, but for this application, the local minimum appears to provide adequate accuracy.

It was found that the same weighting factors would result as long as the taper ratio and dissipation number were held constant. This allows for a look-up table to be used, and only $\beta$ and $\lambda$ are required to find all the parameters. The optimization was performed over a range of dissipation numbers of $10^{-4} \leq \beta \leq 10^{0}$ with 8 points per decade. Providing the boundary value solver any smaller values of $\beta$ resulted in a singular Jacobian within the solver. Adjusting solver tolerances and improving initial guesses provided no solution to this error. The range of taper ratio considered for the optimization was $1 \geq \lambda \geq 0.5$, linearly spaced with 10 points. For this paper, only 6 parameters for $m_{E}$ and $m_{G}$ were considered $(k=6)$; it has been shown that varying the number of parameters has a noticeable effect on accuracy for uniform tubes [3], and the same result is expected here.

A smooth transition between the weighting factors was desired as it allows for interpolation for in-between values of dissipation number and taper ratio. The optimization first selected a value of $\beta$ and performed the optimization for a $\lambda$ value of 1 using the initial guess from $[3,10]$. Once complete, the taper ratio was reduced to the next value $(\lambda=0.95)$, while maintaining the same dissipation number. In order for the series to be smooth, the initial guess parameters for successive iterations were the optimized parameters from the previous $\lambda$. The result of the optimization are 2 three-dimensional matrices of $m_{E}$ and $m_{G}$, and 1 two-dimensional matrix of $\tau$. Two-dimensional interpolation is required to find the weighing factors and propagation time modifier for a given transmission line. 


\section{Frequency Domain Results}

Figure 4 shows the results of the optimization for a dissipation number of $10^{-3}$ and a taper ratio of 0.75 . The $t_{12}$ and $t_{21}$ (anti-diagonal) transfer functions resulting from the TLM are closely matched to the exact solution. The other two functions $t_{11}$ and $t_{22}$ (principal diagonal) are still accurately approximated, however not to the extent of the other antidiagonal terms. As can be seen in $t_{11}$ and $t_{22}$, the first resonant peaks are not perfectly aligned, but all subsequent peaks are matched well. Also to note, the magnitude of the principal diagonal transfer functions are shifted slightly within the exact solution, however, the TLM approximation does not follow this shift. This magnitude shift becomes greater as more taper is added to the model. In all cases, this new model is a better approximation than the previous models given in $[5,6]$.

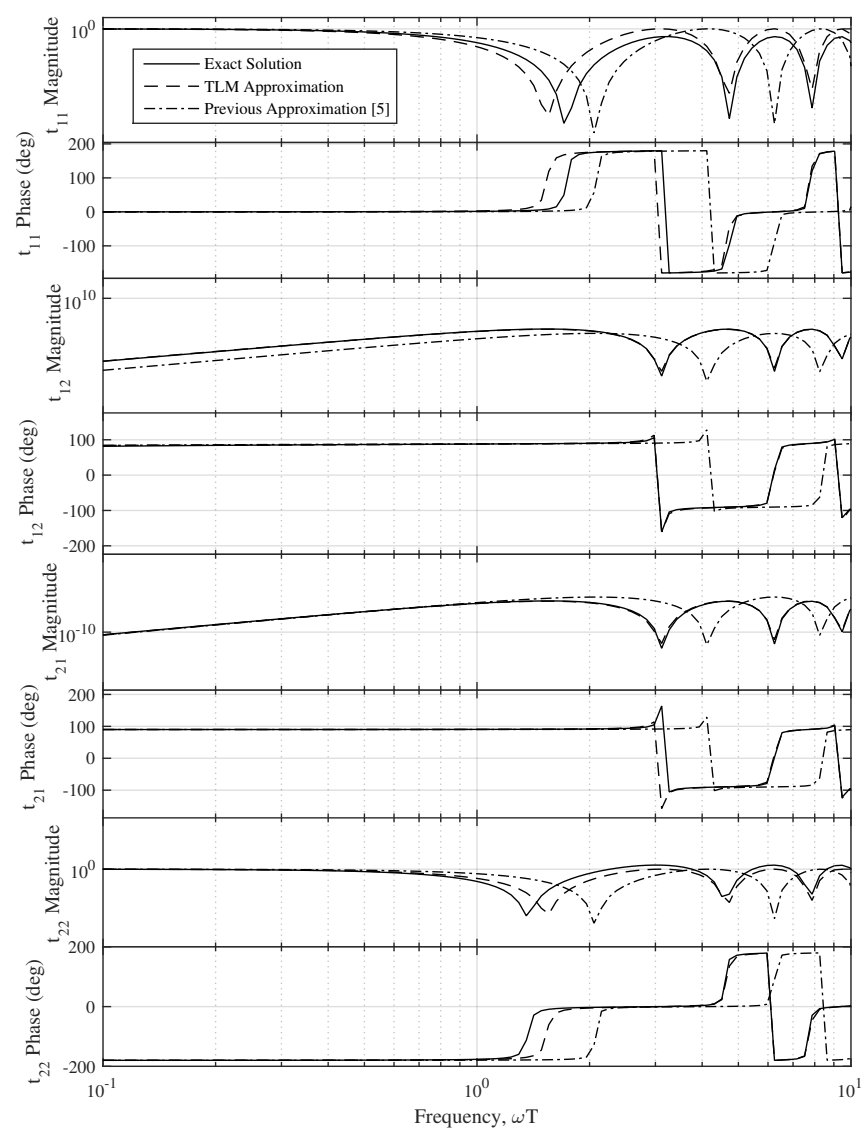

Figure 4: Transmission matrix for $\beta=0.001$ and $\lambda=0.75$.

In order to show some contrast, fig. 5 shows the transmission matrix results for the less extreme case of a larger dissipation number of $10^{-1}$ and a taper ratio of 0.9 . In this case it can be seen that there is a closer approximation of the first resonant peak. Also, it can be seen that the anti-diagonal terms of the transmission matrix are approximated accurately.

Unlike uniform lines of constant cross sectional area, the weighting factors that make up the transmission line model not only vary with dissipation number, $\beta$, but vary also with the taper ratio, $\lambda$. In order to evaluate the accuracy of the model over a range of $\beta$ and $\lambda$, an overall value of error must be defined in order to make relative comparisons. The error

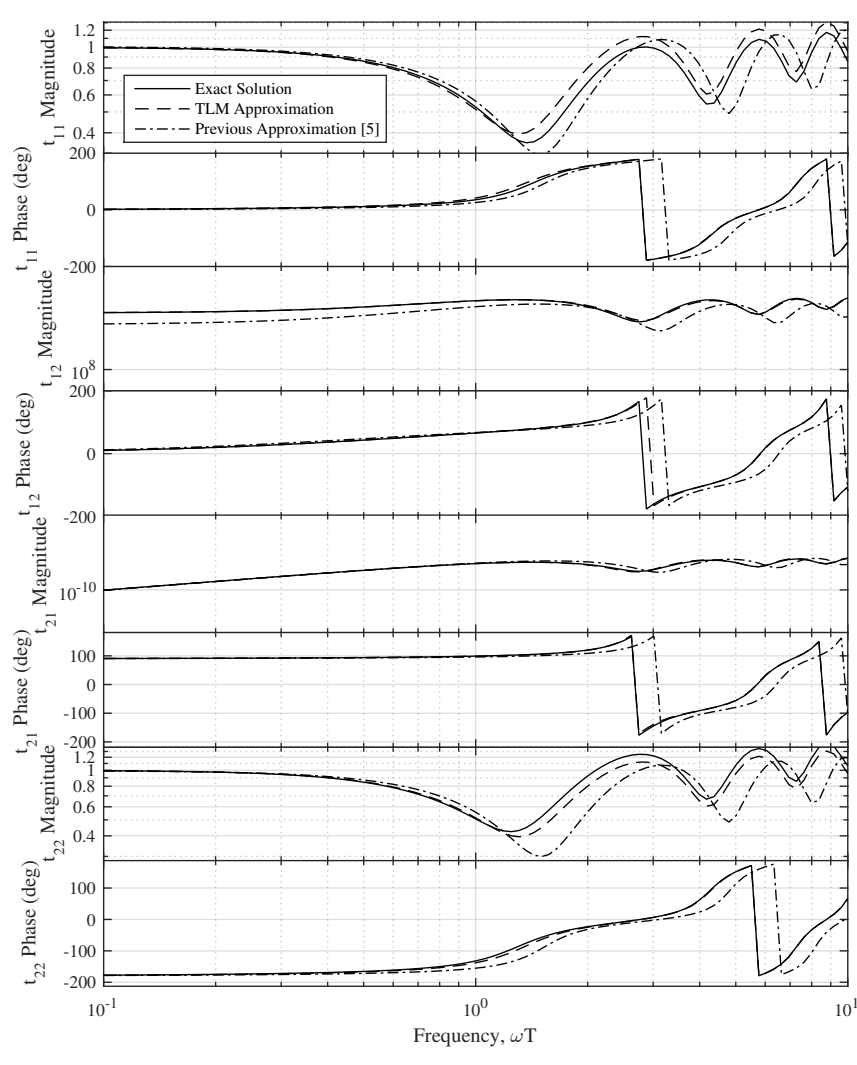

Figure 5: Transmission matrix for $\beta=0.1$ and $\lambda=0.9$.

function indicating the accuracy of fit is given below. This error value was chosen over the optimized minimum calculated from eq. (24) since it is not scaled by frequency. This error value quantifies the exact difference between the magnitude of the transmission matrix terms:

$$
\begin{array}{r}
\varepsilon=\frac{1}{\Omega} \sum_{i=1}^{\Omega}\left[\left|\frac{t_{12}-t_{12}^{*}}{Z_{c}}\right|+\left|\left(t_{21}-t_{21}^{*}\right) Z_{c}\right|\right. \\
+\left|\left(t_{11}-t_{11}^{*}\right)\right| \\
\left.+\left|\left(t_{22}-t_{22}^{*}\right)\right|\right] .
\end{array}
$$

where $\Omega$ is the number of frequency points on which the optimization was performed. The error value in this case is an average of the overall error function. Figure 6 is a visualization of how the error varies with dissipation number and taper ratio. A general observable trend is that the error between the approximated TLM and the exact solution increases with the amount of taper (i.e. decreasing taper ratio) and increases with dissipation number. The optimizations performed in [3] were considered acceptable as long as $\beta \leq 0.5$. So by maintaining the same error from $\lambda=1$ to lower dissipation numbers and taper ratios, an approximately triangular region of acceptance can be found. In order to keep error low, it is recommended to stay within the bounded region as shown in the figure $(\varepsilon<0.5)$.

In order to better demonstrate the improvement this model has over previous tapered transmission line models, consider fig. 7. The same error plot was calculated as previously, except in this case the error was calculated using the approximated 


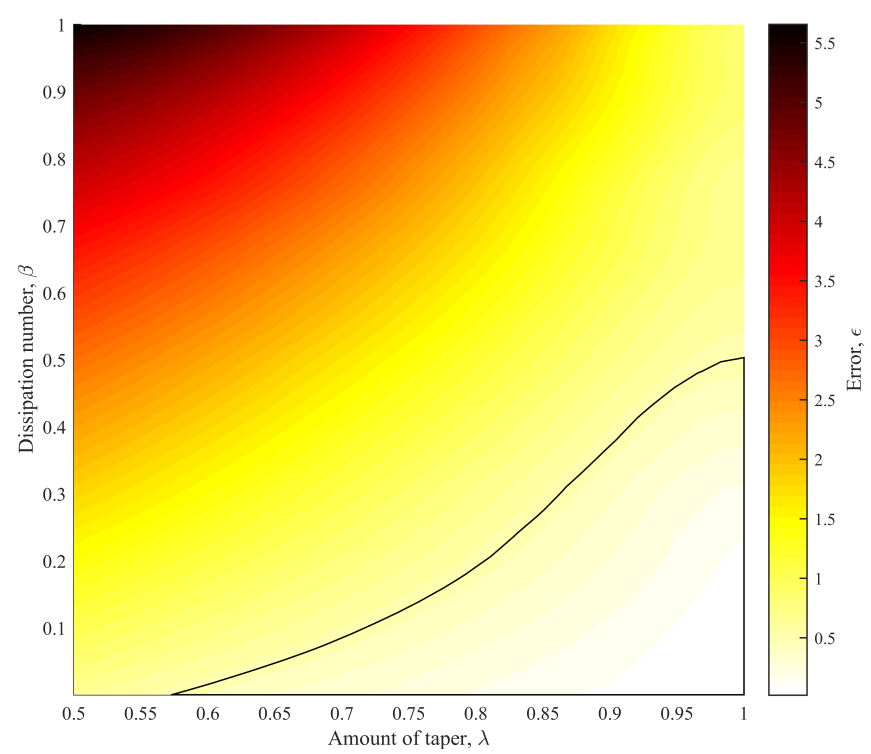

Figure 6: Error analysis for tapered TLM. The black lines show the region of acceptable error, as defined by $\varepsilon<0.5$.

transmission matrix from eq. (5) for comparison to the exact solution. In general, the error is significantly higher, with its scale around 4 times larger than that in fig. 6 . When $\lambda=1$, the approximated transmission matrix analytically becomes the exact solution. This is the reason for the low error region around this value. However, the amount of error increases rapidly with any increase in the amount of taper in the transmission line for the full range of dissipation numbers. Using the same error level as previouly, the enclosed region of acceptability indicated in fig. 7 is considerably smaller. It is also important to note that in its current form eq. (5) cannot be implemented in the time domain. The work performed in [6] approximates eq. (5) for simulations in the time domain. However, being based off this approximated transmission matrix, it can be expected that the error would only be greater than the error shown in fig. 7 .

\section{Time Domain Results}

In order to simulate the proposed model in the time domain, a MATLAB $R$ Simulink $(R$ model was created, available for download at [11]. The overall model can be seen in fig. 8 . The $E, F$, and $G$ transfer functions were implemented using the equations described previously. The $e^{-j \omega T}$ block contains a transport delay of time $T^{\prime}$ which applies the modified wave propagation time to the model. The $E$ transfer function block is shown in fig. 9 to demonstrate how the summation of weighted transfer functions was implemented.

The TLM can be arranged with any combination of inputs and outputs depending on how it needs to fit into a simulation model, and what other models it needs to connect to. For simplicity, only one combination of inputs and outputs is examined here. The inputs to the TLM are inlet flow and outlet pressure, while the outputs are inlet pressure and outlet flow. The outlet pressure was held constant at 0 , and the flow inlet was given a step in flow rate. The simulation arrangement is

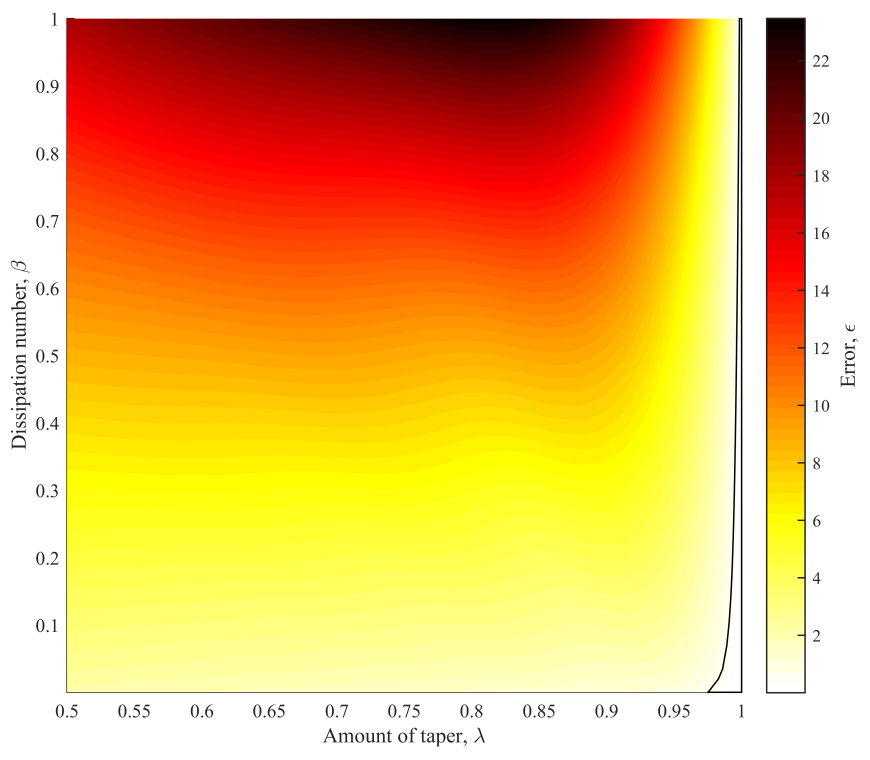

Figure 7: Error analysis for approximate solution from [5]. The black lines show the region of acceptable error, as defined by $\varepsilon<0.5$. Note that the error scale is different than the scale in fig. 6

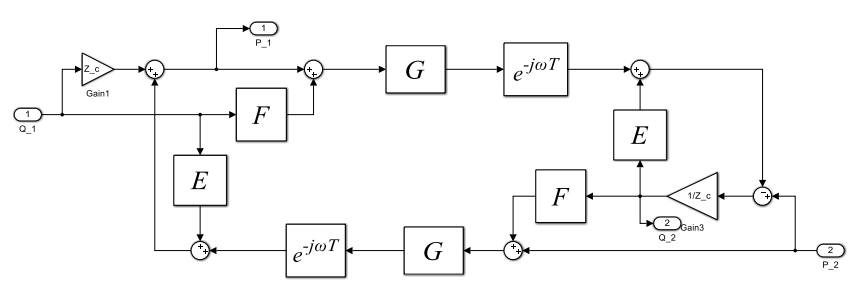

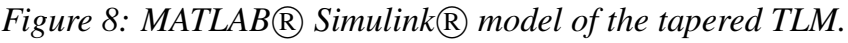

shown in fig. 10 .

Using the same parameters from the transmission matrices computed in fig. 4 and fig. 5, time domain simulations were performed and are shown in fig. 11 and fig. 12 respectively. The solver that was used is MATLAB $R$ 's ode $23 t$ with a relative tolerance of $10^{-6}$. This solver was chosen as it works well for stiff problems such as this, and has shown to solve TLM models relatively quickly when compared to non-stiff solvers such as ode45. The outlet flow and inlet pressure response are shown for the 2 parameter sets. The flow response is normalized through dividing by the step change in flow. In both simulation cases, the flow response oscillations always settled to 1 , thus indicating that the model has proper flow continuity. The inlet pressure response is normalized through dividing by the step change in flow multiplied by the characteristic impedance of the line. The model does appear to respond in a stable manner when a step input is applied. This is an important characteristic as these models are typically used in simulations where high frequencies are present. These simulated responses look as expected, as they are similar to that seen in $[2,3]$. Previously, the TLM has been used to model inertance tubes within switched inertance converters [3], a type of hydraulic circuit that efficiently converts pressure and flow by switching a digital hydraulic valve at very high frequency. 


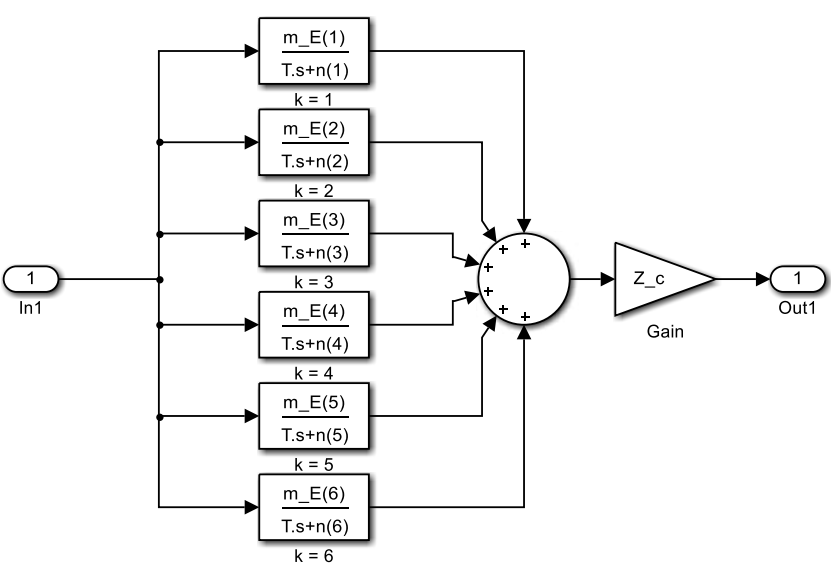

Figure 9: MATLAB $\mathrm{R}$ Simulink $\mathrm{R}$ implementation of the $E$ transfer function.

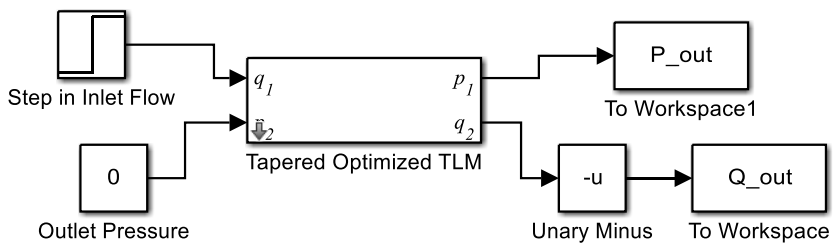

Figure 10: Simulation set-up.

The model presented here is believed to suit well for this area of fluid power research.

\section{Conclusions}

While excellent models exist for modelling fluid dynamics within transmission lines of uniform cross-section, further development in modelling tapered transmission lines was required. This research looks at modelling the fluid dynamics within tapered lines by using the TLM structure and weighted transfer functions. The results of this TLM approximation show significantly improved accuracy over previous tapered transmission line models. This model can be used for a variety of applications where line dynamics must be considered, such as switched inertance converters. A complete

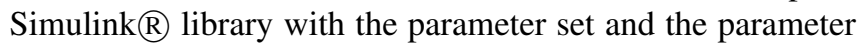

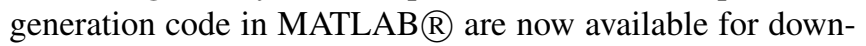
load [11]. Future work on this topic plans to look at performing experiments to validate the proposed model, as well as look at applying the TLM to flexible walled transmission lines. Also, research is planned to investigate the effects of shaped inertance tubes on the performance of switched inertance converters. Transmission lines of arbitrarily changing cross section can be modelled using this approach by successively connecting multiple tapered transmission line segments together. Using shaped inertance tubes has theoretically shown an increase in efficiency over using uniform tubes [8], and by applying this model, higher accuracy and speed in these simulations are expected.

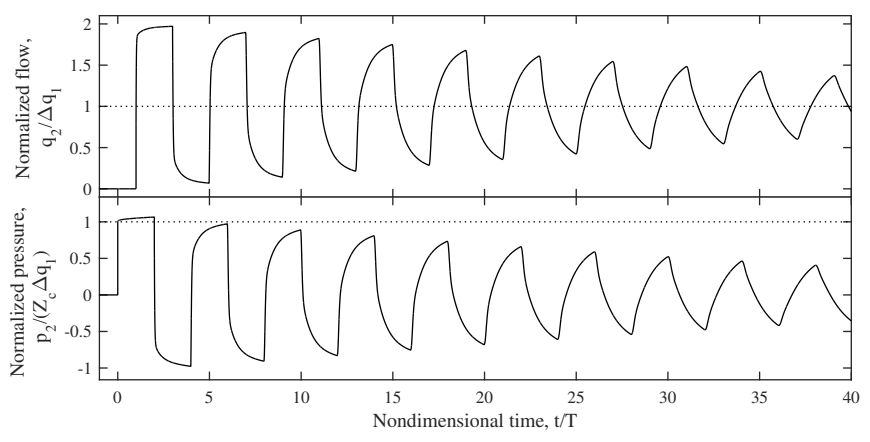

Figure 11: Simulated results for $\beta=0.001$ and $\lambda=0.75$.

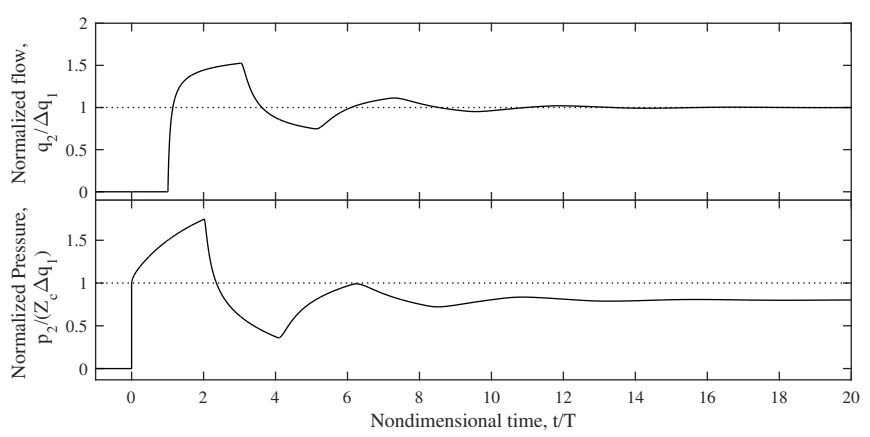

Figure 12: Simulated results for $\beta=0.1$ and $\lambda=0.9$.

\section{References}

[1] P Krus, K Weddfelt, and J O Palmberg. Fast pipeline models for simulation of hydraulic systems. Journal of Dynamic Systems Measurement and Control, 1994.

[2] N Johnston. The transmission line method for modelling laminar flow of liquid in pipelines. Journal of Systems and Control Engineering, 226(5):586-597, 2012.

[3] N Johnston, M Pan, and S Kudzma. An enhanced transmission line method for modelling laminar flow of liquid in pipelines. Journal of Systems and Control Engineering, 228(4):193-206, 2014.

[4] W Zielke. Frequency-dependent friction in transient pipe flow. Journal of Basic Engineering, 90(1):109$115,1968$.

[5] T Muto, Y Kinoshita, and R Yoneda. Dynamic response of tapered fluid lines (1st report, transfer matrix and frequency response). Bulletin of the JSME, 24(191):809$815,1981$.

[6] M Tahmeen, T Muto, and H Yamada. Simulation of dynamic responses of tapered fluid lines. JSME International Journal, Series B, 44(2):247-254, 2001.

[7] T J Viersma. Analysis, synthesis and design of hydraulic servosystems and pipelines. Studies in Mechanical Engineering - Volume 1, 1980.

[8] T Wiens. Analysis and mitigation of valve switching losses in switched inertance converters. In Proceedings 
of the ASME/BATH 2015 Symposium on Fluid Power \& Motion Control, 2015.

[9] P Krus, A Jansson, J Palmberg, and K Weddfelt. Distributed simulation of hydromechanical systems. In Third Bath International Fluid Power Workshop, 1990.

[10] N Johnston.

Simulink models, http://people.bath.ac.uk/ensdnj/models/newtlm.html, 2014.

[11] J ven der Buhs and T Wiens. Transmission line models, https://github.com/tkw954/, 2017.

\section{Nomenclature}

\begin{tabular}{|c|c|c|}
\hline Designation & Denotation & Unit \\
\hline$A(x)$ & $\begin{array}{l}\text { Cross-sectional area of trans- } \\
\text { mission line at point } x\end{array}$ & $\mathrm{~m}^{2}$ \\
\hline$b$ & $\begin{array}{l}E \text { transfer function scaling } \\
\text { factor }\end{array}$ & \\
\hline$C_{1}, C_{2}$ & $\begin{array}{l}\text { Characteristic pressures of the } \\
\text { TLM }\end{array}$ & $\mathrm{Pa}$ \\
\hline$c$ & Local speed of sound & $\mathrm{m} / \mathrm{s}$ \\
\hline$D$ & Dissipation Number [6] & \\
\hline$E, F, G$ & $\begin{array}{l}\text { TLM weighted transfer func- } \\
\text { tions }\end{array}$ & \\
\hline$f$ & Optimization objective function & \\
\hline$J_{0}, J_{2}$ & Bessel functions of the first kind & \\
\hline$j$ & Imaginary designation & \\
\hline$k$ & Number of weighting factors & \\
\hline$l$ & Length of transmission line & $\mathrm{m}$ \\
\hline$m_{E i}, m_{G i}$ & Weighting factors & \\
\hline$N$ & $\begin{array}{l}\text { Frequency-dependent friction } \\
\text { term }\end{array}$ & \\
\hline$n_{i}$ & Weighting factors & \\
\hline$P$ & Fourier transform of pressure & \\
\hline$p$ & Pressure & $\mathrm{Pa}$ \\
\hline$Q$ & Fourier transform of flow & \\
\hline $\bar{q}$ & Flow & $\mathrm{m}^{3} / \mathrm{s}$ \\
\hline$R$ & Steady state resistance & $\mathrm{Pa} \cdot \mathrm{s} / \mathrm{m}^{3}$ \\
\hline$r$ & Pipe radius & $\mathrm{m}$ \\
\hline$S$ & $\begin{array}{l}\text { Normalized Laplace operator } \\
\text { [6] }\end{array}$ & \\
\hline$s$ & Laplace operator & \\
\hline$T$ & Wave propagation time & $\mathrm{s}$ \\
\hline$T^{\prime}$ & $\begin{array}{l}\text { Modified wave propagation } \\
\text { time }\end{array}$ & $\mathrm{s}$ \\
\hline$t$ & Time & $\mathrm{s}$ \\
\hline$t_{11}, t_{12}, t_{21}, t_{22}$ & $\begin{array}{l}\text { Transmission matrix exact } \\
\text { terms }\end{array}$ & \\
\hline$t_{11}^{*}, t_{12}^{*}, t_{21}^{*}, t_{22}^{*}$ & $\begin{array}{l}\text { Transmission matrix approxim- } \\
\text { ated terms }\end{array}$ & \\
\hline$x$ & Axial direction & \\
\hline$Z_{c}$ & Characteristic impedance & $\mathrm{Pa} \cdot \mathrm{s} / \mathrm{m}^{3}$ \\
\hline$\beta$ & Dissipation number & \\
\hline
\end{tabular}

\begin{tabular}{lll}
\hline Designation & Denotation & Unit \\
\hline$\Gamma$ & Propagation operator [6] & \\
$\varepsilon$ & Error & \\
$\theta$ & Taper angle & $\mathrm{rad}$ \\
$\lambda$ & Taper ratio & $\mathrm{m}^{2} / \mathrm{s}$ \\
$\nu$ & Kinematic viscosity & \\
$\xi$ & Convergence/Divergence para- & \\
& meter [6] & $\mathrm{kg} / \mathrm{m}^{3}$ \\
$\rho$ & Fluid density & \\
$\Omega$ & Wave propagation time modifier & \\
& Number of frequency points on & \\
& which the optimization is per- & \\
$\omega$ & formed & $\mathrm{rad} / \mathrm{s}$ \\
\hline
\end{tabular}

